\title{
Comparative study of antiretroviral drug regimens and drug-drug interactions between younger and older HIV-infected patients at a tertiary care teaching hospital in South Korea
}

This article was published in the following Dove Press journal:

Therapeutics and Clinical Risk Management

\author{
Mi Seon Park ${ }^{1, *}$ \\ Young-Mo Yang ${ }^{2, *}$ \\ Ju-Sin Kim' \\ Eun Joo $\mathrm{Choi}^{2}$ \\ 'Department of Pharmacy, Chonbuk \\ National University Hospital, \\ Jeonju, South Korea; ${ }^{2}$ Department \\ of Pharmacy, College of Pharmacy, \\ Chosun University, Gwangju, \\ South Korea \\ *These authors contributed equally \\ to this work
}

Correspondence: Eun Joo Choi Department of Pharmacy, College of Pharmacy, Chosun University, 309

Pilmun-daero, Dong-gu, Gwangju 6I452, South Korea

Tel +82622306382

Fax +82622225414

Email ejchoi@chosun.ac.kr
Background: With the advent of combination antiretroviral therapy (ART), people living with HIV have lived to older age. So they have experienced age-related illnesses and have taken non-antiretroviral (ARV) medications to manage these illnesses. The aims of this study were to investigate the use patterns of ARV agents in HIV-positive patients by age and to evaluate potential or contraindicated drug-drug interactions (DDIs) between ARV and non-ARV.

Methods: This study was retrospectively conducted with HIV-infected patients receiving ART medications between October 2011 and September 2017 at Chonbuk National University Hospital in South Korea. Data were collected by reviewing patients' electronic medical charts.

Results: Among 207 patients diagnosed with HIV infection, 183 (86.9\% males; 104 aged $<50$ years and 79 aged $\geq 50$ years) were selected based on inclusion criteria. In 2017, the most frequently prescribed ART regimen was nucleoside reverse transcriptase inhibitors (NRTIs)/integrase strand transfer inhibitors (INSTIs; total, $66.3 \%$; $<50$ years, $36.3 \%$; $\geq 50$ years, $30.0 \%$ ) followed by NRTIs/protease inhibitors (PIs; total, $23.8 \%$; $<50$ years, $15.0 \%$; $\geq 50$ years, $8.8 \%$ ). In 2017, the most frequently prescribed NRTI combination was abacavir/lamivudine (total, 34.4\%; $<50$ years, $20.6 \%$; $\geq 50$ years, $13.8 \%$ ) followed by tenofovir alafenamide/ emtricitabine (FTC; total, $31.3 \%$; $<50$ years, $16.3 \%$; $\geq 50$ years, $15.0 \%$ ) and tenofovir disoproxil fumarate/FTC (total, 28.1\%; $<50$ years, 16.9\%; $\geq 50$ years, 11.3\%). In 2017, elvitegravir (EVG)/cobicistat (COBI; total, 57.1\%; <50 years, 30.4\%; $\geq 50$ years, $26.8 \%$ ) was most frequently prescribed followed by dolutegravir (total, 32.1\%; $<50$ years, $19.6 \%$; $\geq 50$ years, $12.5 \%$ ). Potential or contraindicated DDIs between boosted PIs with ritonavir or EVG/COBI and coprescribed drugs occurred most frequently.

Conclusion: Currently, NRTIs/INSTIs is the most frequently prescribed ARV combination. Abacavir/lamivudine, tenofovir alafenamide/FTC, and tenofovir disoproxil fumarate/FTC are the most used NRTIs, and EVG/COBI followed by dolutegravir is the most prescribed INSTIs. Potential or contraindicated DDIs occur mainly between boosted PIs or EVG/COBI and nonARV medications.

Keywords: human immunodeficiency virus, highly active antiretroviral therapy, drug utilization, drug interactions, Korea, elderly patients

\section{Introduction}

Since the advent of combination antiretroviral therapy (ART), the survival and quality of life of people living with HIV (PLWH) have steadily improved, thereby increasing the number of older PLWH. ${ }^{1,2}$ A 2013 estimate revealed that $\sim 4.2$ million PLWH 
across the world were $>50$ years old, and it is expected that this number will continue to increase. ${ }^{3}$ An aging population of PLWH is likely to experience age-associated illnesses such as cardiovascular diseases (CVD), cancers, osteoporosis, and cognitive impairment, similar to the general population. These age-related comorbidities may require chronic treatment. Consequently, it is necessary to not only suppress the viral load of HIV but also control age-related, non-AIDS-related illnesses for effectively managing an aging population of PLWH.

The combination of antiretroviral (ARV) and non-ARV medications in an aging population of PLWH may lead to adverse events (AEs), drug-drug interactions (DDIs), and poor drug adherence, all of which have negative effects on the efficacy and safety of ARV and non-ARV medications. ${ }^{4-8}$ In particular, the rate of incidence of DDIs is likely to rise in an aging population of PLWH due to polypharmacy for the treatment of multiple comorbidities along with the HIV infection. ${ }^{4,9-11}$ According to a retrospective clinical study involving HIV-positive patients aged $\geq 50$ years, the average number of total prescribed medications was 14.2 \pm 5.9 , and that of concomitant medications excluding ARVs was 11.6 $\pm 5.7 .^{7}$ Twenty-five contraindicated DDIs were found to occur in 20 (8.1\%) HIV-infected patients. ${ }^{7}$ In other retrospective clinical studies, potential and contraindicated DDIs were found in $71(62.8 \%)$ and $6(5.3 \%)$ patients, respectively, out of 113 HIV-positive patients receiving comedications. ${ }^{9}$

The incidence rate of DDIs in HIV-positive patients may vary according to the ART regimens used. Potential DDIs may occur more frequently in HIV-positive patients on ritonavir (RTV)- or cobicistat (COBI)-boosted protease inhibitor (PI)-based or on non-nucleoside reverse transcriptase inhibitor (NNRTI)-based ART regimens. ${ }^{11,12}$ PI-based ART regimens are nine times as likely to induce potential DDIs as regimens without PIs. ${ }^{13}$ Additionally, patients treated with ART regimens containing NNRTIs were likely to experience about 4.3 times as many potential DDIs as their counterparts. ${ }^{13}$ Therefore, it is important that appropriate ART regimens that are less likely to interact with other comedications and less likely to affect non-AIDS illnesses are used, especially in older HIV-positive patients who are already suffering from comorbidities.

This study was aimed to investigate ARV usage patterns among HIV-positive patients in an age-wise manner and to evaluate the potential or contraindicated DDIs between ARVs and concomitantly prescribed drugs.

\section{Methods}

The Institutional Review Board (IRB) of Chonbuk National University Hospital granted ethical approval for this study
(CUH 2017-11-028). The IRB waived the requirement for obtaining informed consent from the participants in this study since their data were deidentified and anonymously encoded prior to commencing analyses. This study was retrospectively conducted with the following categories of patients visiting Chonbuk National University Hospital, located in the city of Jeonju in North Jeolla Province of South Korea, between October 2011 and September 2017. The inclusion criteria were the following: 1 ) age $\geq 18$ years; 2) diagnosis of HIV infection; and 3) having received ART at least once.

A retrospective chart review of the electronic medical records of selected HIV-infected patients was conducted, in which a trained hospital pharmacist collected the following information from paper case report forms: demographic characteristics (sex, age, weight, height, and body mass index), risk factors for HIV infection, prior HIV treatment, hepatitis B virus and hepatitis $\mathrm{C}$ virus positivity, comorbidities, prescribed medications for HIV infection and other diseases, and laboratory values (HIV-1 RNA copy, CD4+T-cell count, and estimated glomerular filtration rate $[\mathrm{eGFR}])$.

Older adults living with HIV infection may suffer from more comorbidities and experience more rapid physical and cognitive aging than their normal counterparts do. Study of HIV-related literature reveals that the aging HIV-infected population is represented by patients aged $\geq 50$ years. ${ }^{14}$ To compare the differences in the use of ARV drug regimens among individuals of different ages, the selected patients were divided into two groups, namely, patients $<50$ years and patients $\geq 50$ years. In order to assess the usage patterns of HIV regimens in a year-wise manner during the study period, the regimens were categorized on the basis of the combination of two nucleoside reverse transcriptase inhibitors (NRTIs) and a third ARV agent based on recently published HIV treatment guidelines. ${ }^{15,16}$ The potential and contraindicated DDIs between ARV agents and concomitantly prescribed drugs during the study period were also investigated using the Liverpool HIV Drug Interactions website. ${ }^{17}$

All the analyses were conducted using SAS, version 9.3 (SAS Institute Inc., Cary, NC, USA). The mean and SD were used for continuous variables, whereas the frequencies (n) and percentages $(\%)$ were used for the categorical variables. The independent $t$-test was performed for comparing the differences in the means of the continuous variables, and the chi-squared test or Fisher's exact test was also conducted for comparing the differences in the proportions of the categorical variables. $P$-values $<0.05$ were considered to be statistically significant. 


\section{Results}

During the study period, 207 patients were diagnosed with HIV infection, of which 183 patients (104 patients aged $<50$ years and 79 patients aged $\geq 50$ years) who met the aforementioned inclusion criteria were selected for the analysis (Figure 1). The baseline characteristics of the patients are presented in Table 1 and arranged according to their ages. The average age of all the patients was $47.3 \pm 12.4$ years, and most of the patients $(86.9 \%)$ were males. The eGFR levels of the patients aged $<50$ years were significantly higher than those of the patients aged $\geq 50$ years. However, the incidence rates of diabetes mellitus (DM), hypertension (HTN), cancer, and benign prostatic hyperplasia were significantly higher in the patients aged $\geq 50$ years than in patients aged $<50$ years.

The ARV drug combination regimens used during the study period are presented in a year-wise manner in Table 2. In 2011, the combination of NRTIs and PIs was most frequently prescribed in both groups of patients. Between 2011 and 2014, the prescription rate of this combination remained almost stable; however, the prescription rate gradually declined after 2014. The prescription rate of the combination of NRTIs and integrase strand transfer inhibitors (INSTIs) had tended to gradually increase ever since its first use in 2012, and this combination was the most frequently prescribed ARV drug regimen in 2017.

The prescribed ARV drugs are presented in a year-wise manner in Table 3. Although zidovudine (ZDV)/lamivudine (3TC) was most frequently prescribed in 2011 (54.7\%), its prescription rate gradually decreased by $2.5 \%$ in 2017 . The prescription rate of abacavir $(\mathrm{ABC}) / 3 \mathrm{TC}$ remained stable throughout the study period. The prescription rate of tenofovir disoproxil fumarate (TDF)/emtricitabine (FTC) exhibited a steady increase from $2012(31.0 \%)$ to 2016 (60.4\%). However, in 2017, this rate became almost half (28.1\%) of that in 2016 . The prescription rate of tenofovir alafenamide (TAF)/FTC, which was first used in 2017, was $31.3 \%$, which may account for the decrease in the prescription rate of TDF/FTC. Rilpivirine (RPV) had been the most frequently prescribed NNRTI ever since its first use in 2015. Although efavirenz had been the most frequently prescribed NNRTI from 2011 to 2015, its prescription ended in 2017. Atazanavir and lopinavir (LPV)/ RTV had been steadily prescribed until the introduction of elvitegravir (EVG) combined with COBI in 2015. In 2017, the prescription rate of boosted PIs with RTV or COBI was $97.8 \%$. The prescription rates of dolutegravir (DTG) and EVG/COBI have been gradually increasing since their first use in 2015, whereas that of raltegravir (RAL) decreased after 2014.

The classes of drugs coadministered with ARVs are presented in an age-wise manner in Table 4. The drug classes that had significantly higher rates of use in patients aged $\geq 50$ years than in those aged $<50$ years were drugs prescribed for ailments of the alimentary tract and metabolism; dermatologicals; and drugs for diseases of the cardiovascular system, blood and blood forming organs, and the genitourinary system and sex hormones.

Based on the data obtained by using the Liverpool HIV Drug Interactions website, the potential or contraindicated DDIs are summarized in Table 5. A total of 194 potential or contraindicated DDIs were identified, and among them,

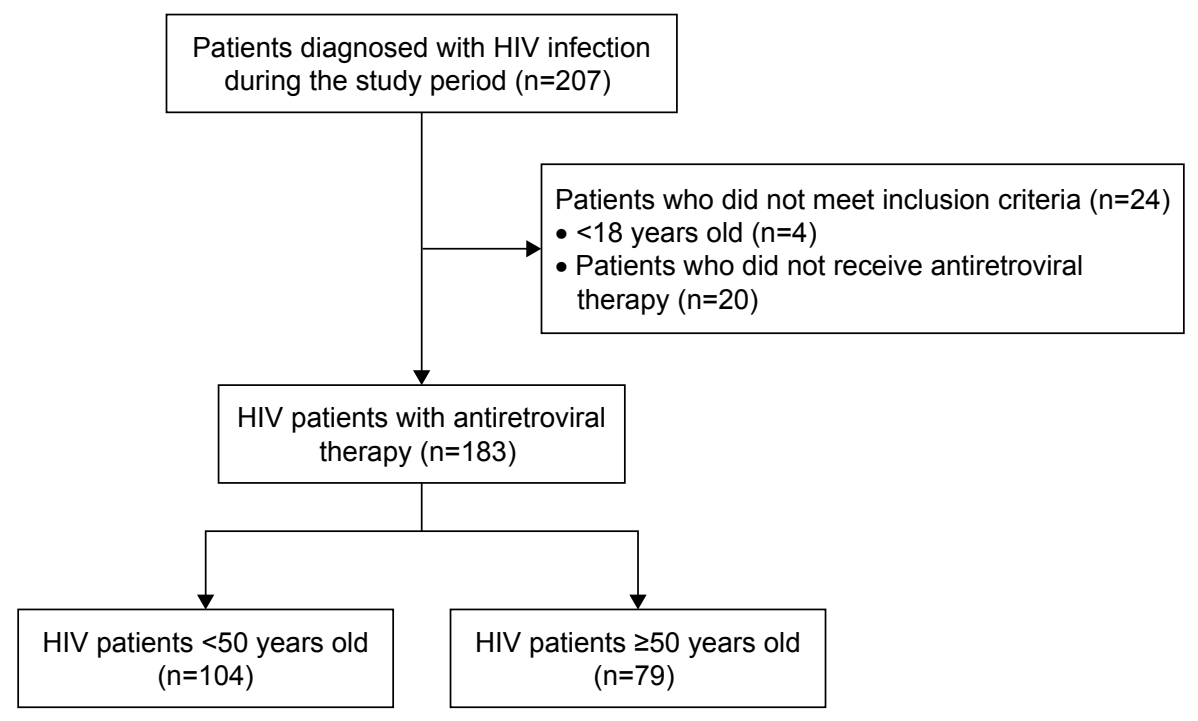

Figure I Flow diagram of steps in the selection of study subjects. 
Table I Baseline characteristics of the patients included in the study

\begin{tabular}{|c|c|c|c|c|}
\hline Variable & $\begin{array}{l}\text { All patients } \\
(n=\mid 83)\end{array}$ & $\begin{array}{l}<50 \text { years } \\
(n=104)\end{array}$ & $\begin{array}{l}\geq 50 \text { years } \\
(n=79)\end{array}$ & $P$-value \\
\hline Age, mean (SD), years & $47.3(12.4)$ & $38.7(7.8)$ & $58.5(7.2)$ & $<0.001$ \\
\hline \multicolumn{5}{|l|}{ Sex, n (\%) } \\
\hline Male & $159(86.9)$ & $89(85.6)$ & $70(88.6)$ & \\
\hline Female & $24(13.1)$ & $15(14.4)$ & $9(11.4)$ & 0.547 \\
\hline BMI, mean (SD), kg/m² & $22.3(3.4)$ & $21.7(3.5)$ & $23.1(3.2)$ & 0.056 \\
\hline \multicolumn{5}{|l|}{ Risk factors for HIV infection, n (\%) } \\
\hline Intravenous drug use & $\mathrm{I}(0.5)$ & $\mathrm{I}(\mathrm{I} .0)$ & $0(0.0)$ & \\
\hline Hetero/homosexual & $37(20.2)$ & $19(18.3)$ & $18(22.8)$ & 0.522 \\
\hline Unknown & $145(79.2)$ & $84(80.8)$ & $61(77.2)$ & \\
\hline \multicolumn{5}{|l|}{ Previous HIV treatment, n (\%) } \\
\hline Naïve & $108(59.0)$ & $66(63.5)$ & $42(53.2)$ & \\
\hline Experienced & $75(4 \mid .0)$ & $38(36.5)$ & $37(46.8)$ & 0.161 \\
\hline \multirow{2}{*}{\multicolumn{5}{|c|}{ HIV-I RNA copy, n (\%) }} \\
\hline & & & & \\
\hline HIV-I RNA copy $\geq 100,000$ copies $/ \mathrm{mL}$ & $4 I(23.2)$ & $30(29.4)$ & II (14.7) & 0.026 \\
\hline CD4+T-cell count, mean (SD), cells $/ \mathrm{mm}^{3}$ & $316.3(223.3)$ & $296.9(220.6)$ & $343.2(225.6)$ & 0.175 \\
\hline \multicolumn{5}{|l|}{ CD4+T-cell count, cells $/ \mathrm{mm}^{3}, \mathrm{n}(\%)$} \\
\hline$<50$ & $27(15.3)$ & $18(17.6)$ & $9(12.2)$ & \\
\hline$\geq 50$ to $<200$ & $33(18.8)$ & $19(18.6)$ & $14(18.9)$ & 0.601 \\
\hline$\geq 200$ & $116(65.9)$ & $65(63.7)$ & $51(68.9)$ & \\
\hline HBV positive, n (\%) & II (6.0) & $4(3.7)$ & $7(8.9)$ & 0.212 \\
\hline HCV positive, n (\%) & $I(0.5)$ & $\mathrm{I}(\mathrm{I} .0)$ & $0(0.0)$ & 1.000 \\
\hline eGFR, mean (SD), $\mathrm{mL} / \mathrm{min} / \mathrm{I} .73 \mathrm{~m}^{2}$ & $108.0(20.2)$ & $113.0(2 \mid .2)$ & $100.5(16.2)$ & 0.004 \\
\hline \multicolumn{5}{|l|}{ Comorbidity, n (\%) } \\
\hline Diabetes mellitus & $28(15.3)$ & $6(5.8)$ & $22(27.8)$ & $<0.001$ \\
\hline Hypertension & $10(5.5)$ & $I(1.0)$ & $9(11.4)$ & 0.003 \\
\hline Dyslipidemia & $4(2.2)$ & $\mathrm{I}(\mathrm{I} .0)$ & $3(3.8)$ & 0.317 \\
\hline Cancer & $15(8.2)$ & $4(3.8)$ & $11(13.9)$ & 0.014 \\
\hline Asthma & $3(1.6)$ & $\mathrm{I}(\mathrm{I} .0)$ & $2(2.5)$ & 0.579 \\
\hline Chronic obstructive pulmonary disease & $\mathrm{I}(0.5)$ & $0(0.0)$ & $\mathrm{I}(\mathrm{I} .3)$ & 0.432 \\
\hline Dementia, cognitive impairment & $5(2.7)$ & $2(1.9)$ & $3(3.8)$ & 0.653 \\
\hline Chronic kidney disease & $4(2.2)$ & $3(2.9)$ & $\mathrm{I}(\mathrm{I} .3)$ & 0.635 \\
\hline Benign prostatic hyperplasia & $9(4.9)$ & $0(0.0)$ & $9(11.4)$ & $<0.001$ \\
\hline Erectile dysfunction & $4(2.2)$ & $2(1.9)$ & $2(2.5)$ & 1.000 \\
\hline Myelodysplastic syndromes & $\mathrm{I}(0.5)$ & $0(0.0)$ & $\mathrm{I}(\mathrm{l} .3)$ & 0.432 \\
\hline Gastritis, gastroesophageal reflux disease & $17(9.3)$ & $7(6.7)$ & $10(12.7)$ & 0.171 \\
\hline Thyroid disease & $\mathrm{I}(0.5)$ & $0(0.0)$ & $\mathrm{I}(\mathrm{l} .3)$ & 0.432 \\
\hline Seizure & $4(2.2)$ & $4(3.8)$ & $0(0.0)$ & 0.135 \\
\hline Stroke & $7(3.8)$ & $2(1.9)$ & $5(6.3)$ & 0.242 \\
\hline Acute coronary syndrome & $2(1.1)$ & $\mathrm{I}(\mathrm{I} .0)$ & $\mathrm{I}(\mathrm{I} .3)$ & 1.000 \\
\hline Depression & $8(4.4)$ & $4(3.8)$ & $4(5.1)$ & 1.000 \\
\hline \multicolumn{5}{|l|}{ Opportunistic infections, n (\%) } \\
\hline Syphilis & $29(15.8)$ & I5 (I4.4) & $14(17.7)$ & 0.545 \\
\hline Pneumocystis pneumonia & $23(12.6)$ & $14(13.5)$ & 9 (II.4) & 0.676 \\
\hline Candidiasis & $22(12.0)$ & $15(14.4)$ & $7(8.9)$ & 0.252 \\
\hline Varicella-Zoster virus & $14(7.7)$ & $9(8.7)$ & $5(6.3)$ & 0.558 \\
\hline Tuberculosis & $9(4.9)$ & $5(4.8)$ & $4(5.1)$ & 0.937 \\
\hline Cytomegalovirus & $9(4.9)$ & $6(5.8)$ & $3(3.8)$ & 0.541 \\
\hline Human papillomavirus & $9(4.9)$ & $5(4.8)$ & $4(5.1)$ & 0.937 \\
\hline Pneumonia & $9(4.9)$ & $4(3.8)$ & $5(6.3)$ & 0.442 \\
\hline Herpes simplex virus & $8(4.4)$ & $6(5.8)$ & $2(2.5)$ & 0.289 \\
\hline Mycobacterium avium complex & $4(2.2)$ & $3(2.9)$ & $\mathrm{I}(\mathrm{I} .3)$ & 0.458 \\
\hline Cryptococcosis & $4(2.2)$ & $2(1.9)$ & $2(2.5)$ & 0.780 \\
\hline $\mathrm{JC}$ virus & $2(1.1)$ & $2(1.9)$ & $0(0.0)$ & 0.218 \\
\hline Toxoplasmic encephalitis & $2(1.1)$ & $0(0.0)$ & $2(2.5)$ & 0.103 \\
\hline Kaposi's sarcoma & $\mathrm{I}(0.5)$ & $\mathrm{I}(\mathrm{I} .0)$ & $0(0.0)$ & 0.382 \\
\hline \multicolumn{5}{|l|}{ ARV regimens, $n(\%)$} \\
\hline NRTIs/NNRTIs & $37(20.2)$ & $21(20.2)$ & $16(20.3)$ & \\
\hline NRTIs/Pls & $100(54.6)$ & $60(57.7)$ & $40(50.6)$ & 0.649 \\
\hline NRTIs/INSTIs & $43(23.5)$ & $21(20.2)$ & $22(27.8)$ & \\
\hline NRTIs/NNRTIs/Pls & $3(1.6)$ & $2(1.9)$ & $\mathrm{I}(\mathrm{I} .3)$ & \\
\hline
\end{tabular}

Abbreviations: ARV, antiretroviral; BMI, body mass index; eGFR, estimated glomerular filtration rate; HBV, hepatitis B virus; HCV, hepatitis C virus; INSTIs, integrase strand transfer inhibitors; NNRTls, non-nucleoside reverse transcriptase inhibitors; NRTIs, nucleoside reverse transcriptase inhibitors; Pls, protease inhibitors. 
Table 2 Antiretroviral drug combination regimens used during the study period

\begin{tabular}{|c|c|c|c|c|c|c|c|c|}
\hline \multirow[t]{2}{*}{ Regimen } & \multirow[t]{2}{*}{ Age, years } & \multicolumn{7}{|c|}{ Year, n (\%) } \\
\hline & & $\begin{array}{l}2011 \\
(n=53)\end{array}$ & $\begin{array}{l}2012 \\
(n=71)\end{array}$ & $\begin{array}{l}2013 \\
(n=89)\end{array}$ & $\begin{array}{l}2014 \\
(n=|| 5)\end{array}$ & $\begin{array}{l}2015 \\
(n=130)\end{array}$ & $\begin{array}{l}2016 \\
(n=154)\end{array}$ & $\begin{array}{l}2017 \\
(n=160)\end{array}$ \\
\hline NRTIs/NNRTIs & $\begin{array}{l}\text { Total } \\
<50 \\
\geq 50\end{array}$ & $\begin{array}{l}9(17.0) \\
5(9.4) \\
4(7.5)\end{array}$ & $\begin{array}{l}\text { II (15.5) } \\
6(8.5) \\
5(7.0)\end{array}$ & $\begin{array}{l}8(9.0) \\
3(3.4) \\
5(5.6)\end{array}$ & $\begin{array}{l}13(1 \mathrm{I} .3) \\
5(4.3) \\
8(7.0)\end{array}$ & $\begin{array}{l}\text { II (8.5) } \\
4(3.1) \\
7(5.4)\end{array}$ & $\begin{array}{l}23(14.9) \\
11(7.1) \\
12(7.8)\end{array}$ & $\begin{array}{l}8(5.0) \\
3(1.9) \\
5(3.1)\end{array}$ \\
\hline NRTIs/Pls & $\begin{array}{l}\text { Total } \\
<50 \\
\geq 50\end{array}$ & $\begin{array}{l}40(75.5) \\
23(43.4) \\
17(32.1)\end{array}$ & $\begin{array}{l}50(70.4) \\
30(42.3) \\
20(28.2)\end{array}$ & $\begin{array}{l}61(68.5) \\
36(40.5) \\
25(28.1)\end{array}$ & $\begin{array}{l}76(66.1) \\
45(39.1) \\
31(27.0)\end{array}$ & $\begin{array}{l}67(51.5) \\
39(30.0) \\
28(21.5)\end{array}$ & $\begin{array}{l}64(4 I .6) \\
40(26.0) \\
24(15.6)\end{array}$ & $\begin{array}{l}38(23.8) \\
24(15.0) \\
14(8.8)\end{array}$ \\
\hline NRTIs/INSTIs & $\begin{array}{l}\text { Total } \\
<50 \\
\geq 50\end{array}$ & $\begin{array}{l}- \\
- \\
-\end{array}$ & $\begin{array}{l}7(9.9) \\
- \\
7(9.9)\end{array}$ & $\begin{array}{l}17(19.1) \\
5(5.6) \\
12(13.5)\end{array}$ & $\begin{array}{l}23(20.0) \\
7(6.1) \\
16(13.9)\end{array}$ & $\begin{array}{l}45(34.6) \\
20(15.4) \\
25(19.2)\end{array}$ & $\begin{array}{l}59(38.3) \\
30(19.5) \\
29(18.8)\end{array}$ & $\begin{array}{l}106(66.3) \\
58(36.3) \\
48(30.0)\end{array}$ \\
\hline NRTIs/NNRTIs/Pls & $\begin{array}{l}\text { Total } \\
<50 \\
\geq 50\end{array}$ & $\begin{array}{l}3(5.7) \\
2(3.8) \\
1(1.9)\end{array}$ & $\begin{array}{l}- \\
- \\
-\end{array}$ & $\begin{array}{l}- \\
- \\
-\end{array}$ & $\begin{array}{l}- \\
- \\
-\end{array}$ & $\begin{array}{l}- \\
- \\
-\end{array}$ & $\begin{array}{l}- \\
- \\
-\end{array}$ & $\begin{array}{l}- \\
- \\
-\end{array}$ \\
\hline NRTIs/PIs/INSTIs & $\begin{array}{l}\text { Total } \\
<50 \\
\geq 50\end{array}$ & $\begin{array}{l}- \\
- \\
-\end{array}$ & $\begin{array}{l}- \\
- \\
-\end{array}$ & $\begin{array}{l}I(I . I) \\
- \\
I(I . I)\end{array}$ & $\begin{array}{l}\text { I (0.9) } \\
- \\
\text { I }(0.9)\end{array}$ & $\begin{array}{l}2(1.5) \\
\text { I }(0.8) \\
I(0.8)\end{array}$ & $\begin{array}{l}2(1.3) \\
\text { I }(0.6) \\
I(0.6)\end{array}$ & $\begin{array}{l}\text { I }(0.6) \\
- \\
\text { I }(0.6)\end{array}$ \\
\hline $\begin{array}{l}\text { NRTIs/NNRTIs/ } \\
\text { INSTIs }\end{array}$ & $\begin{array}{l}\text { Total } \\
<50 \\
\geq 50\end{array}$ & $\begin{array}{l}- \\
- \\
-\end{array}$ & $\begin{array}{l}- \\
- \\
-\end{array}$ & $\begin{array}{l}- \\
- \\
-\end{array}$ & $\begin{array}{l}- \\
- \\
-\end{array}$ & $\begin{array}{l}- \\
- \\
-\end{array}$ & $\begin{array}{l}- \\
- \\
-\end{array}$ & $\begin{array}{l}\text { I }(0.6) \\
- \\
\text { I }(0.6)\end{array}$ \\
\hline Non-NRTIs & $\begin{array}{l}\text { Total } \\
<50 \\
\geq 50\end{array}$ & $\begin{array}{l}\text { I (I.9) } \\
\text { I (I.9) } \\
-\end{array}$ & $\begin{array}{l}3(4.2) \\
2(2.8) \\
1(1.4)\end{array}$ & $\begin{array}{l}2(2.2) \\
2(2.2) \\
-\end{array}$ & $\begin{array}{l}2(1.7) \\
2(1.7) \\
-\end{array}$ & $\begin{array}{l}5(3.8) \\
3(2.3) \\
2(1.5)\end{array}$ & $\begin{array}{l}6(3.9) \\
3(1.9) \\
3(1.9)\end{array}$ & $\begin{array}{l}6(3.8) \\
3(1.9) \\
3(1.9)\end{array}$ \\
\hline
\end{tabular}

Abbreviations: INSTIs, integrase strand transfer inhibitors; NNRTIs, non-nucleoside reverse transcriptase inhibitors; NRTIs, nucleoside reverse transcriptase inhibitors; Pls, protease inhibitors.

Table 3 NRTIs, NNRTIs, Pls, and INSTIs used during the study period

\begin{tabular}{|c|c|c|c|c|c|c|c|c|}
\hline \multirow{2}{*}{$\begin{array}{l}\text { Antiretroviral } \\
\text { drug }\end{array}$} & \multirow[t]{2}{*}{ Age, years } & \multicolumn{7}{|c|}{ Year, n (\%) } \\
\hline & & 2011 & 2012 & 2013 & 2014 & 2015 & 2016 & 2017 \\
\hline NRTIs & & $(n=53)$ & $(n=7 I)$ & $(n=89)$ & $(n=I \mid 5)$ & $(n=130)$ & $(n=154)$ & $(n=160)$ \\
\hline $\mathrm{ABC} / 3 \mathrm{TC}$ & $\begin{array}{l}\text { Total } \\
<50 \\
\geq 50\end{array}$ & $\begin{array}{l}12(22.6) \\
8(15.1) \\
4(7.5)\end{array}$ & $\begin{array}{l}\text { I8 (25.4) } \\
\text { II (I5.5) } \\
7(9.9)\end{array}$ & $\begin{array}{l}28(31.5) \\
16(18.0) \\
12(13.5)\end{array}$ & $\begin{array}{l}40(34.8) \\
22(19.1) \\
18(15.7)\end{array}$ & $\begin{array}{l}42(32.3) \\
22(16.9) \\
20(15.4)\end{array}$ & $\begin{array}{l}50(32.5) \\
28(18.2) \\
22(14.3)\end{array}$ & $\begin{array}{l}55(34.4) \\
33(20.6) \\
22(13.8)\end{array}$ \\
\hline TDF/FTC & $\begin{array}{l}\text { Total } \\
<50 \\
\geq 50\end{array}$ & $\begin{array}{l}- \\
- \\
-\end{array}$ & $\begin{array}{l}22(31.0) \\
10(14.1) \\
12(16.9)\end{array}$ & $\begin{array}{l}32(36.0) \\
16(18.0) \\
16(18.0)\end{array}$ & $\begin{array}{l}46(40.0) \\
24(20.9) \\
22(19.1)\end{array}$ & $\begin{array}{l}77(59.2) \\
39(30.0) \\
38(29.2)\end{array}$ & $\begin{array}{l}93(60.4) \\
52(33.8) \\
41(26.6)\end{array}$ & $\begin{array}{l}45(28.1) \\
27(16.9) \\
18(11.3)\end{array}$ \\
\hline ZDV/3TC & $\begin{array}{l}\text { Total } \\
<50 \\
\geq 50\end{array}$ & $\begin{array}{l}29(54.7) \\
17(32.1) \\
12(22.6)\end{array}$ & $\begin{array}{l}28(39.4) \\
15(21.1) \\
13(18.3)\end{array}$ & $\begin{array}{l}27(31.5) \\
12(13.5) \\
15(18.0)\end{array}$ & $\begin{array}{l}27(23.5) \\
\text { II }(9.6) \\
16(13.9)\end{array}$ & $\begin{array}{l}7(5.4) \\
4(3.1) \\
3(2.3)\end{array}$ & $\begin{array}{l}5(3.2) \\
2(1.3) \\
3(1.9)\end{array}$ & $\begin{array}{l}4(2.5) \\
1(0.6) \\
3(1.9)\end{array}$ \\
\hline $3 \mathrm{TC} / \mathrm{d} 4 \mathrm{~T}$ & $\begin{array}{l}\text { Total } \\
<50 \\
\geq 50\end{array}$ & $\begin{array}{l}7(13.2) \\
2(3.8) \\
5(9.4)\end{array}$ & $\begin{array}{l}- \\
- \\
-\end{array}$ & $\begin{array}{l}- \\
- \\
-\end{array}$ & $\begin{array}{l}- \\
- \\
-\end{array}$ & $\begin{array}{l}- \\
- \\
-\end{array}$ & $\begin{array}{l}- \\
- \\
-\end{array}$ & $\begin{array}{l}- \\
- \\
-\end{array}$ \\
\hline TAF/FTC & $\begin{array}{l}\text { Total } \\
<50 \\
\geq 50\end{array}$ & $\begin{array}{l}- \\
- \\
-\end{array}$ & $\begin{array}{l}- \\
- \\
-\end{array}$ & $\begin{array}{l}- \\
- \\
-\end{array}$ & $\begin{array}{l}- \\
- \\
-\end{array}$ & $\begin{array}{l}- \\
- \\
-\end{array}$ & $\begin{array}{l}- \\
- \\
-\end{array}$ & $\begin{array}{l}50(31.3) \\
26(16.3) \\
24(15.0)\end{array}$ \\
\hline $3 \mathrm{TC} / \mathrm{ddl}$ & $\begin{array}{l}\text { Total } \\
<50 \\
\geq 50\end{array}$ & $\begin{array}{l}\text { I (I.9) } \\
\text { I (I.9) } \\
-\end{array}$ & $\begin{array}{l}- \\
- \\
-\end{array}$ & $\begin{array}{l}- \\
- \\
-\end{array}$ & $\begin{array}{l}- \\
- \\
-\end{array}$ & $\begin{array}{l}- \\
- \\
-\end{array}$ & $\begin{array}{l}- \\
- \\
-\end{array}$ & $\begin{array}{l}- \\
- \\
-\end{array}$ \\
\hline 3TC & $\begin{array}{l}\text { Total } \\
<50 \\
\geq 50\end{array}$ & $\begin{array}{l}3(5.7) \\
2(3.8) \\
1(1.9)\end{array}$ & $\begin{array}{l}- \\
- \\
-\end{array}$ & $\begin{array}{l}- \\
- \\
-\end{array}$ & - & - & $\begin{array}{l}- \\
- \\
-\end{array}$ & $\begin{array}{l}- \\
- \\
-\end{array}$ \\
\hline
\end{tabular}


Table 3 (Continued)

\begin{tabular}{|c|c|c|c|c|c|c|c|c|}
\hline \multirow{2}{*}{$\begin{array}{l}\text { Antiretroviral } \\
\text { drug }\end{array}$} & \multirow[t]{2}{*}{ Age, years } & \multicolumn{7}{|c|}{ Year, n (\%) } \\
\hline & & 2011 & 2012 & 2013 & 2014 & 2015 & 2016 & 2017 \\
\hline NRTIs & & $(n=53)$ & $(n=7 I)$ & $(n=89)$ & $(n=\mid 15)$ & $(n=130)$ & $(n=154)$ & $(n=160)$ \\
\hline Not used & $\begin{array}{l}\text { Total } \\
<50 \\
\geq 50\end{array}$ & $\begin{array}{l}\text { I (I.9) } \\
\text { I (I.9) } \\
-\end{array}$ & \begin{tabular}{|l|}
$3(4.2)$ \\
$2(2.8)$ \\
$1(1.4)$
\end{tabular} & $\begin{array}{l}2(2.2) \\
2(2.2) \\
-\end{array}$ & $\begin{array}{l}2(1.7) \\
2(1.7) \\
-\end{array}$ & $\begin{array}{l}4(3.1) \\
2(1.5) \\
2(1.5)\end{array}$ & $\begin{array}{l}6(3.9) \\
3(1.9) \\
3(1.9)\end{array}$ & $\begin{array}{l}6(3.8) \\
3(1.9) \\
3(1.9)\end{array}$ \\
\hline NNRTIs & & $(n=13)$ & $(n=14)$ & $(n=10)$ & $(n=15)$ & $(n=15)$ & $(n=28)$ & $(n=14)$ \\
\hline EFV & $\begin{array}{l}\text { Total } \\
<50 \\
\geq 50\end{array}$ & $\begin{array}{l}9(69.2) \\
5(38.5) \\
4(30.8)\end{array}$ & $\begin{array}{l}\text { II (78.6) } \\
6(42.9) \\
5(35.7)\end{array}$ & $\begin{array}{l}8(88.9) \\
3(30.0) \\
5(50.0)\end{array}$ & \begin{tabular}{|l|}
$13(86.7)$ \\
$5(33.3)$ \\
$8(53.3)$
\end{tabular} & $\begin{array}{l}10(66.7) \\
4(26.7) \\
6(40.0)\end{array}$ & $\begin{array}{l}2(7.1) \\
\text { I (3.6) } \\
\text { I (3.6) }\end{array}$ & $\begin{array}{l}- \\
- \\
-\end{array}$ \\
\hline ETR & $\begin{array}{l}\text { Total } \\
<50 \\
\geq 50\end{array}$ & $\begin{array}{l}\text { I (7.7) } \\
\text { I (7.7) } \\
-\end{array}$ & $\begin{array}{l}3(2 \mid .4) \\
2(14.3) \\
I(7 . I)\end{array}$ & $\begin{array}{l}2(20.0) \\
- \\
-\end{array}$ & $\begin{array}{l}2(13.3) \\
- \\
-\end{array}$ & $\begin{array}{l}4(26.7) \\
3(20.0) \\
I(6.7)\end{array}$ & $\begin{array}{l}6(21.4) \\
3(10.7) \\
3(10.7)\end{array}$ & $\begin{array}{l}6(42.9) \\
3(21.4) \\
3(21.4)\end{array}$ \\
\hline NVP & $\begin{array}{l}\text { Total } \\
<50 \\
\geq 50\end{array}$ & $\begin{array}{l}3(23.1) \\
2(15.4) \\
1(7.7)\end{array}$ & $\begin{array}{l}- \\
- \\
-\end{array}$ & $\begin{array}{l}- \\
- \\
-\end{array}$ & $\begin{array}{l}- \\
- \\
-\end{array}$ & $\begin{array}{l}- \\
- \\
-\end{array}$ & $\begin{array}{l}- \\
- \\
-\end{array}$ & $\begin{array}{l}- \\
- \\
-\end{array}$ \\
\hline RPV & $\begin{array}{l}\text { Total } \\
<50 \\
\geq 50\end{array}$ & $\begin{array}{l}- \\
- \\
-\end{array}$ & $\begin{array}{l}- \\
- \\
-\end{array}$ & $\begin{array}{l}- \\
- \\
-\end{array}$ & $\begin{array}{l}- \\
- \\
-\end{array}$ & $\begin{array}{l}\text { I (6.7) } \\
- \\
\text { I (6.7) }\end{array}$ & $\begin{array}{l}20(71.4) \\
10(35.7) \\
10(35.7)\end{array}$ & $\begin{array}{l}8(57.1) \\
4(28.6) \\
4(28.6)\end{array}$ \\
\hline PIs & & $(n=44)$ & $(n=53)$ & $(n=64)$ & $(n=79)$ & $(n=74)$ & $(n=72)$ & $(n=45)$ \\
\hline ATV & $\begin{array}{l}\text { Total } \\
<50 \\
\geq 50\end{array}$ & $\begin{array}{l}13(29.5) \\
5(11.4) \\
8(18.2)\end{array}$ & $\begin{array}{l}17(32.1) \\
9(17.0) \\
8(15.1)\end{array}$ & $\begin{array}{l}19(29.7) \\
10(15.6) \\
9(14.1)\end{array}$ & $\begin{array}{l}17(2 \mid .5) \\
9(11.4) \\
8(10.1)\end{array}$ & $\begin{array}{l}6(8.1) \\
3(4.1) \\
3(4.1)\end{array}$ & $\begin{array}{l}3(4.2) \\
1(1.4) \\
2(2.8)\end{array}$ & $\begin{array}{l}\text { I (2.2) } \\
\text { I (2.2) } \\
-\end{array}$ \\
\hline ATV/RTV & $\begin{array}{l}\text { Total } \\
<50 \\
\geq 50\end{array}$ & $\begin{array}{l}- \\
- \\
-\end{array}$ & $\begin{array}{l}- \\
- \\
-\end{array}$ & $\begin{array}{l}- \\
- \\
-\end{array}$ & $\begin{array}{l}7(8.9) \\
4(5.1) \\
3(3.8) \\
\end{array}$ & $\begin{array}{l}6(8.1) \\
5(6.8) \\
1(1.4)\end{array}$ & $\begin{array}{l}4(5.6) \\
4(5.6) \\
-\end{array}$ & $\begin{array}{l}\text { I (2.2) } \\
\text { I (2.2) } \\
-\end{array}$ \\
\hline ATV/COBI & $\begin{array}{l}\text { Total } \\
<50 \\
\geq 50\end{array}$ & $\begin{array}{l}- \\
- \\
-\end{array}$ & $\begin{array}{l}- \\
- \\
-\end{array}$ & $\begin{array}{l}- \\
- \\
-\end{array}$ & $\begin{array}{l}- \\
- \\
-\end{array}$ & $\begin{array}{l}- \\
- \\
-\end{array}$ & $\begin{array}{l}- \\
- \\
-\end{array}$ & $\begin{array}{l}2(4.4) \\
\text { I (2.2) } \\
\text { I (2.2) }\end{array}$ \\
\hline LPV/RTV & $\begin{array}{l}\text { Total } \\
<50 \\
\geq 50\end{array}$ & $\begin{array}{l}27(61.4) \\
18(40.9) \\
9(20.5)\end{array}$ & $\begin{array}{l}30(56.6) \\
19(35.8) \\
11(20.8)\end{array}$ & $\begin{array}{l}39(60.9) \\
24(37.5) \\
15(23.4)\end{array}$ & $\begin{array}{l}45(57.0) \\
27(34.2) \\
18(22.8)\end{array}$ & $\begin{array}{l}35(47.3) \\
19(25.7) \\
16(21.6)\end{array}$ & $\begin{array}{l}33(45.8) \\
19(26.4) \\
14(19.4)\end{array}$ & $\begin{array}{l}30(66.7) \\
17(37.8) \\
13(28.9)\end{array}$ \\
\hline DRV/RTV & $\begin{array}{l}\text { Total } \\
<50 \\
\geq 50\end{array}$ & $\begin{array}{l}\text { I (2.3) } \\
\text { I (2.3) } \\
-\end{array}$ & $\begin{array}{l}4(7.5) \\
3(5.7) \\
1(1.9) \\
\end{array}$ & $\begin{array}{l}4(6.3) \\
3(4.7) \\
1(1.6)\end{array}$ & $\begin{array}{l}9 \text { (II.4) } \\
7 \text { (8.9) } \\
2 \text { (2.5) }\end{array}$ & $\begin{array}{l}26(35.1) \\
16(21.6) \\
10(13.5)\end{array}$ & $\begin{array}{l}32(44.4) \\
20(27.8) \\
12(16.7)\end{array}$ & $\begin{array}{l}7(15.6) \\
6(13.3) \\
I(2.2)\end{array}$ \\
\hline $\mathrm{DRV} / \mathrm{COBI}$ & $\begin{array}{l}\text { Total } \\
<50 \\
\geq 50\end{array}$ & $\begin{array}{l}- \\
- \\
-\end{array}$ & $\begin{array}{l}- \\
- \\
-\end{array}$ & $\begin{array}{l}- \\
- \\
-\end{array}$ & $\begin{array}{l}- \\
- \\
-\end{array}$ & $\begin{array}{l}- \\
- \\
-\end{array}$ & $\begin{array}{l}- \\
- \\
-\end{array}$ & $\begin{array}{l}4(8.9) \\
2(4.4) \\
2(4.4)\end{array}$ \\
\hline IDV & $\begin{array}{l}\text { Total } \\
<50 \\
\geq 50\end{array}$ & $\begin{array}{l}3(6.8) \\
2(4.5) \\
I(2.3)\end{array}$ & $\begin{array}{l}2(3.8) \\
\text { I (1.9) } \\
\text { I (1.9) }\end{array}$ & $\begin{array}{l}2(3.1) \\
I(1.6) \\
I(1.6)\end{array}$ & $\begin{array}{l}I(1.3) \\
- \\
I(1.3)\end{array}$ & $\begin{array}{l}- \\
- \\
-\end{array}$ & $\begin{array}{l}- \\
- \\
-\end{array}$ & $\begin{array}{l}- \\
- \\
-\end{array}$ \\
\hline DRV & $\begin{array}{l}\text { Total } \\
<50 \\
\geq 50\end{array}$ & $\begin{array}{l}- \\
- \\
-\end{array}$ & $\begin{array}{l}- \\
- \\
-\end{array}$ & $\begin{array}{l}- \\
- \\
-\end{array}$ & $\begin{array}{l}- \\
- \\
-\end{array}$ & $\begin{array}{l}\text { I (I.4) } \\
- \\
\text { I (I.4) }\end{array}$ & $\begin{array}{l}- \\
- \\
-\end{array}$ & $\begin{array}{l}- \\
- \\
-\end{array}$ \\
\hline INSTIs & & $(n=I)$ & $(n=10)$ & $(n=20)$ & $(n=26)$ & $(n=5 I)$ & $(n=65)$ & $(n=\mid I 2)$ \\
\hline DTG & $\begin{array}{l}\text { Total } \\
<50 \\
\geq 50\end{array}$ & $\begin{array}{l}- \\
- \\
-\end{array}$ & $\begin{array}{l}- \\
- \\
-\end{array}$ & $\begin{array}{l}- \\
- \\
-\end{array}$ & $\begin{array}{l}- \\
- \\
-\end{array}$ & $\begin{array}{l}\text { I (2.0) } \\
\text { I (2.0) } \\
-\end{array}$ & $\begin{array}{l}11(16.9) \\
8(12.3) \\
3(4.6)\end{array}$ & $\begin{array}{l}36(32.1) \\
22(19.6) \\
14(12.5)\end{array}$ \\
\hline EVG/COBI & $\begin{array}{l}\text { Total } \\
<50 \\
\geq 50\end{array}$ & $\begin{array}{l}- \\
- \\
-\end{array}$ & $\begin{array}{l}- \\
- \\
-\end{array}$ & $\begin{array}{l}- \\
- \\
-\end{array}$ & $\begin{array}{l}- \\
- \\
-\end{array}$ & $\begin{array}{l}20(39.2) \\
12(23.5) \\
8(15.7)\end{array}$ & $\begin{array}{l}30(46.2) \\
18(27.7) \\
12(18.5)\end{array}$ & $\begin{array}{l}64(57.1) \\
34(30.4) \\
30(26.8)\end{array}$ \\
\hline RAL & $\begin{array}{l}\text { Total } \\
<50 \\
\geq 50\end{array}$ & $\begin{array}{l}\text { I }(100.0) \\
\text { I }(100.0) \\
-\end{array}$ & $\begin{array}{l}10(100.0) \\
2(20.0) \\
8(80.0)\end{array}$ & $\begin{array}{l}20(100.0) \\
7(35.0) \\
13(65.0)\end{array}$ & $\begin{array}{l}26(100.0) \\
9(34.6) \\
17(65.4)\end{array}$ & $\begin{array}{l}30(58.8) \\
11(21.6) \\
19(37.3)\end{array}$ & $\begin{array}{l}24(36.9) \\
8(12.3) \\
16(24.6)\end{array}$ & $\begin{array}{l}12(10.7) \\
7(6.3) \\
5(4.5)\end{array}$ \\
\hline
\end{tabular}

Abbreviations: 3TC, lamivudine; ABC, abacavir; ATV, atazanavir; COBI, cobicistat; d4T, stavudine; ddl, didanosine; DRV, darunavir; DTG, dolutegravir; EFV, efavirenz; ETR, etravirine; EVG, elvitegravir; FTC, emtricitabine; IDV, indinavir; INSTIs, integrase strand transfer inhibitors; LPV, lopinavir; NNRTIs, nonnucleoside reverse transcriptase inhibitors; NRTIs, nucleoside reverse transcriptase inhibitors; NVP, nevirapine; Pls, protease inhibitors; RAL, raltegravir; RPV, rilpivirine; RTV, ritonavir; TAF, tenofovir alafenamide; TDF, tenofovir disoproxil fumarate; ZDV, zidovudine. 
Table 4 Coadministered drug classes with antiretrovirals among the patients included in the study

\begin{tabular}{|c|c|c|c|c|}
\hline$\overline{\text { Class }}$ & $\begin{array}{l}\text { All patients, } \\
n(\%) \\
(n=I 83)\end{array}$ & $\begin{array}{l}<\mathbf{5 0} \text { years, } \\
\mathrm{n}(\%) \\
(\mathrm{n}=104)\end{array}$ & $\begin{array}{l}\geq \mathbf{5 0} \text { years, } \\
\mathrm{n}(\%) \\
(\mathrm{n}=79)\end{array}$ & $P$-value \\
\hline Alimentary tract and metabolism & $105(57.4)$ & $48(46.2)$ & $57(72.2)$ & $<0.001$ \\
\hline Anti-infectives for systemic use & $77(42.1)$ & $45(43.3)$ & $32(40.5)$ & 0.708 \\
\hline Dermatologicals & $49(26.8)$ & $20(19.2)$ & $29(36.7)$ & 0.008 \\
\hline Musculoskeletal system & $47(25.7)$ & $28(26.9)$ & $19(24.1)$ & 0.660 \\
\hline Nervous system & $47(25.7)$ & $23(22.1)$ & $24(30.4)$ & 0.205 \\
\hline Respiratory system & $44(24.0)$ & $23(22.1)$ & $21(26.6)$ & 0.484 \\
\hline Cardiovascular system & $43(23.5)$ & $18(17.3)$ & $25(31.6)$ & 0.023 \\
\hline Systemic hormonal preparations, excluding sex hormones & $22(12.0)$ & $13(12.5)$ & $9(11.4)$ & 0.819 \\
\hline Blood and blood forming organs & $20(10.9)$ & $7(6.7)$ & $13(16.5)$ & 0.037 \\
\hline Genitourinary system and sex hormones & $18(9.8)$ & $5(4.8)$ & $13(16.5)$ & 0.009 \\
\hline Antineoplastic and immunomodulating agents & $10(5.5)$ & $7(6.7)$ & $3(3.8)$ & 0.518 \\
\hline Sensory organs & $7(3.2)$ & $3(2.9)$ & $4(5.1)$ & 0.467 \\
\hline Antiparasitic products, insecticides, and repellents & $2(1.1)$ & $\mathrm{I}(\mathrm{I} .0)$ & $\mathrm{I}(\mathrm{I} .3)$ & 0.845 \\
\hline
\end{tabular}

$12(6.2 \%)$ contraindicated DDIs were found. Contraindicated DDIs occurred most frequently between boosted PIs with RTV or EVG/COBI and coprescribed drugs such as alfuzosin, clopidogrel, quetiapine, rifampicin, simvastatin, and phenytoin.

\section{Discussion}

In this study, the usage patterns of ARV regimens in HIVpositive patients were studied in an age-wise manner and potential or contraindicated DDIs between ARV and nonARV drugs were investigated. Recently, the most frequently prescribed regimen involved treatment with NRTIs/INSTIs followed by therapy with NRTIs/PIs. ABC/3TC, TDF/ FTC, and TAF/FTC among the NRTIs, LPV/RTV among the PIs, and EVG/COBI and DTG among the INSTIs were frequently prescribed. In addition, DDIs between boosted PIs with RTV or EVG/COBI and coprescribed drugs occurred most frequently.

Numerous diseases such as osteoporosis, DM, chronic liver disease, chronic kidney disease, and CVDs have higher rates of occurrence in the aging population of PLWH than in their HIV-uninfected counterparts. ${ }^{18-20}$ Therefore, in order to effectively manage health conditions in the aging population of PLWH, it is not only essential to suppress the viral load of HIV and allow the immune system to recover but also to control age-associated, non-AIDS illnesses. However, the combined use of ARV and non-ARV drugs is likely to cause various drug-related problems such as DDIs.

The coformulation of ZDV and 3TC was a preferred choice for the treatment of HIV infections in 2011, but it was rarely used in 2017. Instead of this NRTI combination, tenofovir-based combinations (TDF/FTC and TAF/FTC) or the $\mathrm{ABC} / 3 \mathrm{TC}$ combination was usually administered. This trend may be due to several reasons as stated hereafter. The prolonged use of ZDV can lead to more severe adverse reactions such as hematological toxicities (anemia and/or neutropenia) and symptomatic myopathy. ${ }^{21} \mathrm{ZDV} / 3 \mathrm{TC}$ requires twice-daily dosing for effectively suppressing the levels of HIV RNA, whereas the TDF/FTC, TAF/FTC, and $\mathrm{ABC} / 3 \mathrm{TC}$ combinations require once-daily dosing, which may reduce the pill burden for $\mathrm{HIV}$-infected patients and improve their medication adherence rates. ${ }^{21-26}$ Furthermore, it was reported that the efficacy of the ZDV/3TC combination was less robust than TDF/FTC-based ART in achieving viral suppression. ${ }^{27,28}$

The most frequently prescribed regimen in 2017 was NRTIs/INSTIs followed by NRTIs/PIs. Specifically, TDF/ FTC/COBI/EVG (Stribild ${ }^{\circledR}$ ) and TAF/FTC/COBI/EVG $\left(\right.$ Genvoya $\left.^{\circledR}\right)$, approved by the US Food and Drug Administration (FDA) in 2012 and 2015, respectively, consisted of two NRTIs and one INSTI with one booster. ${ }^{24,25}$ The ABC/3TC/DTG (Triumeq ${ }^{\circledR}$ ) combination, consisting of two NRTIs and one INSTI, was approved by the FDA in $2014 .^{26}$ This tendency might have appeared owing to the use of the once-daily, single-tablet regimens as the initial therapy for treatment-naïve patients and switching to simplified, less toxic regimens for treatment-experienced patients..$^{29,30}$

Until 2016, Stribild had been the only tenofovir-based combination; however, in 2017, Genvoya was introduced in the hospital where this study was conducted. As shown in Table 3, the prescription rate of TDF/FTC, the two NRTIs in Stribild, in 2017 (28.1\%) was almost half of 
Table 5 Potential or contraindicated drug-drug interactions between antiretrovirals and other drugs coprescribed with antiretrovirals

\begin{tabular}{|c|c|c|c|c|}
\hline Antiretroviral drug & Comedication & $\begin{array}{l}\text { Frequency, n (\%) } \\
(n=194)\end{array}$ & $\begin{array}{l}\text { Strength of } \\
\text { recommendation }^{\mathrm{a}}\end{array}$ & Quality of evidence \\
\hline \multicolumn{5}{|l|}{ NRTIs } \\
\hline 3TC & Amphotericin & $\mathrm{I}(0.5)$ & Potential interaction & Very low \\
\hline TDF & $\begin{array}{l}\text { Acyclovir } \\
\text { Celecoxib } \\
\text { Clarithromycin } \\
\text { Ganciclovir } \\
\text { Naproxen } \\
\text { Nimesulide } \\
\text { Pentamidine } \\
\text { Topiramate } \\
\text { Verapamil }\end{array}$ & $\begin{array}{l}5(2.6) \\
2(1.0) \\
1(0.5) \\
4(2.1) \\
3(1.5) \\
6(3.1) \\
1(0.5) \\
1(0.5) \\
I(0.5)\end{array}$ & $\begin{array}{l}\text { Potential interaction } \\
\text { Potential interaction } \\
\text { Potential interaction } \\
\text { Potential interaction } \\
\text { Potential interaction } \\
\text { Potential interaction } \\
\text { Potential interaction } \\
\text { Potential interaction } \\
\text { Potential interaction }\end{array}$ & $\begin{array}{l}\text { Very low } \\
\text { Very low } \\
\text { Very low } \\
\text { Very low } \\
\text { Very low } \\
\text { Very low } \\
\text { Very low } \\
\text { Very low } \\
\text { Very low }\end{array}$ \\
\hline ZDV & $\begin{array}{l}\text { Fluconazole } \\
\text { Trimethoprim/ } \\
\text { sulfamethoxazole }\end{array}$ & $\begin{array}{l}2(1.0) \\
1(0.5)\end{array}$ & $\begin{array}{l}\text { Potential interaction } \\
\text { Potential interaction }\end{array}$ & $\begin{array}{l}\text { Low } \\
\text { Low }\end{array}$ \\
\hline \multicolumn{5}{|l|}{ NNRTIs } \\
\hline EFV & $\begin{array}{l}\text { Amlodipine } \\
\text { Moxifloxacin } \\
\text { Nimesulide } \\
\text { Zolpidem }\end{array}$ & $\begin{array}{l}\text { I }(0.5) \\
\text { I }(0.5) \\
\text { I }(0.5) \\
\text { I }(0.5)\end{array}$ & $\begin{array}{l}\text { Potential interaction } \\
\text { Potential interaction } \\
\text { Potential interaction } \\
\text { Potential interaction }\end{array}$ & $\begin{array}{l}\text { Very low } \\
\text { Very low } \\
\text { Very low } \\
\text { Very low }\end{array}$ \\
\hline ETR & $\begin{array}{l}\text { Clarithromycin } \\
\text { Fluconazole } \\
\text { Glimepiride } \\
\text { Lercanidipine } \\
\text { Naproxen } \\
\text { Oxycodone } \\
\text { Rifampicin } \\
\text { Sildenafil } \\
\text { Tamsulosin } \\
\end{array}$ & $\begin{array}{l}I(0.5) \\
2(I .0) \\
2(I .0) \\
I(0.5) \\
I(0.5) \\
I(0.5) \\
I(0.5) \\
I(0.5) \\
I(0.5)\end{array}$ & $\begin{array}{l}\text { Potential interaction } \\
\text { Potential interaction } \\
\text { Potential interaction } \\
\text { Potential interaction } \\
\text { Potential interaction } \\
\text { Potential interaction } \\
\text { Do not coadminister } \\
\text { Potential interaction } \\
\text { Potential interaction }\end{array}$ & $\begin{array}{l}\text { Moderate } \\
\text { Low } \\
\text { Very low } \\
\text { Very low } \\
\text { Very low } \\
\text { Very low } \\
\text { Moderate } \\
\text { High } \\
\text { Very low }\end{array}$ \\
\hline RPV & $\begin{array}{l}\text { Diltiazem } \\
\text { Famotidine } \\
\text { Fluconazole } \\
\text { Itraconazole }\end{array}$ & $\begin{array}{l}\text { I }(0.5) \\
2(1.0) \\
\text { I }(0.5) \\
\text { I }(0.5)\end{array}$ & $\begin{array}{l}\text { Potential interaction } \\
\text { Potential interaction } \\
\text { Potential interaction } \\
\text { Potential interaction }\end{array}$ & $\begin{array}{l}\text { Very low } \\
\text { Low } \\
\text { Very low } \\
\text { Very low }\end{array}$ \\
\hline \multicolumn{5}{|l|}{ Pls } \\
\hline$\overline{\text { ATV }}$ & $\begin{array}{l}\text { Atovaquone/proguanil } \\
\text { Buspirone } \\
\text { Clarithromycin } \\
\text { Escitalopram } \\
\text { Famotidine } \\
\text { Lansoprazole } \\
\text { Nortriptyline } \\
\text { Prednisolone } \\
\text { Rifabutin } \\
\text { Tamsulosin } \\
\text { Zolpidem } \\
\end{array}$ & $\begin{array}{l}\text { I }(0.5) \\
\text { I }(0.5) \\
\text { I }(0.5) \\
\text { I }(0.5) \\
2(I .0) \\
\text { I }(0.5) \\
2(1.0) \\
\text { I }(0.5) \\
\text { I }(0.5) \\
\text { I }(0.5) \\
\text { I }(0.5) \\
\end{array}$ & $\begin{array}{l}\text { Potential interaction } \\
\text { Potential interaction } \\
\text { Potential interaction } \\
\text { Potential interaction } \\
\text { Potential interaction } \\
\text { Do not coadminister } \\
\text { Potential interaction } \\
\text { Potential interaction } \\
\text { Potential interaction } \\
\text { Potential interaction } \\
\text { Potential interaction } \\
\end{array}$ & $\begin{array}{l}\text { Moderate } \\
\text { Very low } \\
\text { Low } \\
\text { Very low } \\
\text { Low } \\
\text { Low } \\
\text { Very low } \\
\text { Very low } \\
\text { High } \\
\text { Very low } \\
\text { Very low } \\
\end{array}$ \\
\hline ATV/COBI & Atorvastatin & $\mathrm{I}(0.5)$ & Potential interaction & Very low \\
\hline ATV/RTV & $\begin{array}{l}\text { Atorvastatin } \\
\text { Clopidogrel } \\
\text { Methylprednisolone }\end{array}$ & $\begin{array}{l}2(1.0) \\
I(0.5) \\
I(0.5) \\
\end{array}$ & $\begin{array}{l}\text { Potential interaction } \\
\text { Do not coadminister } \\
\text { Potential interaction }\end{array}$ & $\begin{array}{l}\text { Very low } \\
\text { Low } \\
\text { Very low }\end{array}$ \\
\hline LPV/RTV & $\begin{array}{l}\text { Alfuzosin } \\
\text { Alprazolam } \\
\text { Amlodipine } \\
\text { Azithromycin } \\
\text { Atorvastatin }\end{array}$ & $\begin{array}{l}\text { I }(0.5) \\
I(0.5) \\
3(1.5) \\
I(0.5) \\
8(4.1)\end{array}$ & $\begin{array}{l}\text { Do not coadminister } \\
\text { Potential interaction } \\
\text { Potential interaction } \\
\text { Potential interaction } \\
\text { Potential interaction }\end{array}$ & $\begin{array}{l}\text { Moderate } \\
\text { Very low } \\
\text { Very low } \\
\text { Very low } \\
\text { High }\end{array}$ \\
\hline
\end{tabular}


Table 5 (Continued)

\begin{tabular}{|c|c|c|c|c|}
\hline Antiretroviral drug & Comedication & $\begin{array}{l}\text { Frequency, } n(\%) \\
(n=194)\end{array}$ & $\begin{array}{l}\text { Strength of } \\
\text { recommendation }\end{array}$ & Quality of evidence ${ }^{b}$ \\
\hline & $\begin{array}{l}\text { Clarithromycin } \\
\text { Clindamycin } \\
\text { Estradiol } \\
\text { Fentanyl } \\
\text { Gliclazide } \\
\text { Glimepiride } \\
\text { Hydroxyzine } \\
\text { Lacidipine } \\
\text { Methylprednisolone } \\
\text { Mirtazapine } \\
\text { Nortriptyline } \\
\text { Nifedipine } \\
\text { Oxcarbazepine } \\
\text { Quetiapine } \\
\text { Rifabutin } \\
\text { Rifampicin } \\
\text { Risperidone } \\
\text { Sildenafil } \\
\text { Simvastatin } \\
\text { Tadalafil } \\
\text { Tamsulosin } \\
\text { Trazodone } \\
\text { Valproate } \\
\text { Voriconazole } \\
\text { Zolpidem }\end{array}$ & $\begin{array}{l}3(I .5) \\
\text { I }(0.5) \\
\text { I }(0.5) \\
2(I .0) \\
\text { I }(0.5) \\
4(2.1) \\
3(I .5) \\
\text { I }(0.5) \\
2(I .0) \\
2(I .0) \\
2(1.0) \\
\text { I }(0.5) \\
\text { I }(0.5) \\
\text { I }(0.5) \\
\text { I }(0.5) \\
\text { I }(0.5) \\
\text { I }(0.5) \\
\text { I }(0.5) \\
\text { I }(0.5) \\
2(I .0) \\
\text { I }(0.5) \\
\text { I }(0.5) \\
\text { I }(0.5) \\
\text { I }(0.5) \\
6(3.1)\end{array}$ & $\begin{array}{l}\text { Potential interaction } \\
\text { Potential interaction } \\
\text { Potential interaction } \\
\text { Potential interaction } \\
\text { Potential interaction } \\
\text { Potential interaction } \\
\text { Potential interaction } \\
\text { Potential interaction } \\
\text { Potential interaction } \\
\text { Potential interaction } \\
\text { Potential interaction } \\
\text { Potential interaction } \\
\text { Potential interaction } \\
\text { Do not coadminister } \\
\text { Potential interaction } \\
\text { Do not coadminister } \\
\text { Potential interaction } \\
\text { Potential interaction } \\
\text { Do not coadminister } \\
\text { Potential interaction } \\
\text { Potential interaction } \\
\text { Potential interaction } \\
\text { Potential interaction } \\
\text { Potential interaction } \\
\text { Potential interaction }\end{array}$ & $\begin{array}{l}\text { Very low } \\
\text { Very low } \\
\text { Very low } \\
\text { Very low } \\
\text { Very low } \\
\text { Very low } \\
\text { Very low } \\
\text { Very low } \\
\text { Very low } \\
\text { Very low } \\
\text { Very low } \\
\text { Very low } \\
\text { Very low } \\
\text { Very low } \\
\text { High } \\
\text { High } \\
\text { Very low } \\
\text { High } \\
\text { Moderate } \\
\text { High } \\
\text { Very low } \\
\text { Moderate } \\
\text { Moderate } \\
\text { Moderate } \\
\text { Very low }\end{array}$ \\
\hline DRV/RTV & $\begin{array}{l}\text { Atorvastatin } \\
\text { Atovaquone/proguanil } \\
\text { Clarithromycin } \\
\text { Clopidogrel } \\
\text { Colchicine } \\
\text { Glimepiride } \\
\text { Hydrocortisone } \\
\text { Hydroxyzine } \\
\text { Itraconazole } \\
\text { Methylprednisolone } \\
\text { Nifedipine } \\
\text { Oxycodone } \\
\text { Prednisolone } \\
\text { Quetiapine } \\
\text { Rifabutin } \\
\text { Sildenafil } \\
\text { Valproate } \\
\text { Zolpidem }\end{array}$ & $\begin{array}{l}\text { 3 }(I .5) \\
\text { I }(0.5) \\
\text { I }(0.5) \\
\text { I }(0.5) \\
\text { I }(0.5) \\
\text { I }(0.5) \\
\text { I }(0.5) \\
\text { 2 }(I .0) \\
\text { I }(0.5) \\
\text { I }(0.5) \\
\text { I }(0.5) \\
\text { I }(0.5) \\
2(I .0) \\
\text { I }(0.5) \\
\text { I }(0.5) \\
\text { I }(0.5) \\
\text { I }(0.5) \\
3(I .5)\end{array}$ & $\begin{array}{l}\text { Potential interaction } \\
\text { Potential interaction } \\
\text { Potential interaction } \\
\text { Do not coadminister } \\
\text { Potential interaction } \\
\text { Potential interaction } \\
\text { Potential interaction } \\
\text { Potential interaction } \\
\text { Potential interaction } \\
\text { Potential interaction } \\
\text { Potential interaction } \\
\text { Potential interaction } \\
\text { Potential interaction } \\
\text { Do not coadminister } \\
\text { Potential interaction } \\
\text { Potential interaction } \\
\text { Potential interaction } \\
\text { Potential interaction }\end{array}$ & $\begin{array}{l}\text { High } \\
\text { Very low } \\
\text { Moderate } \\
\text { Low } \\
\text { Very low } \\
\text { Very low } \\
\text { Very low } \\
\text { Very low } \\
\text { Very low } \\
\text { Very low } \\
\text { Very low } \\
\text { Very low } \\
\text { Very low } \\
\text { Very low } \\
\text { Low } \\
\text { Very low } \\
\text { Very low } \\
\text { Very low }\end{array}$ \\
\hline IDV & Glimepiride & $\mathrm{I}(0.5)$ & Potential interaction & Very low \\
\hline \multicolumn{5}{|l|}{ INSTIs } \\
\hline DTG & $\begin{array}{l}\text { Magnesium } \\
\text { Metformin }\end{array}$ & $\begin{array}{l}\text { I (0.5) } \\
5(2.6)\end{array}$ & $\begin{array}{l}\text { Potential interaction } \\
\text { Potential interaction }\end{array}$ & $\begin{array}{l}\text { Very low } \\
\text { Low }\end{array}$ \\
\hline EVG/COBI & $\begin{array}{l}\text { Alprazolam } \\
\text { Amlodipine } \\
\text { Atorvastatin } \\
\text { Clonazepam } \\
\text { Dexamethasone } \\
\text { Fentanyl } \\
\text { Glimepiride } \\
\text { Hydroxyzine }\end{array}$ & $\begin{array}{l}\text { I }(0.5) \\
2(I .0) \\
\text { I }(0.5) \\
\text { I }(0.5) \\
\text { I }(0.5) \\
\text { I }(0.5) \\
\text { I }(0.5) \\
\text { I }(0.5)\end{array}$ & $\begin{array}{l}\text { Potential interaction } \\
\text { Potential interaction } \\
\text { Potential interaction } \\
\text { Potential interaction } \\
\text { Potential interaction } \\
\text { Potential interaction } \\
\text { Potential interaction } \\
\text { Potential interaction }\end{array}$ & $\begin{array}{l}\text { Very low } \\
\text { Very low } \\
\text { Very low } \\
\text { Very low } \\
\text { Very low } \\
\text { Very low } \\
\text { Very low } \\
\text { Very low }\end{array}$ \\
\hline
\end{tabular}


Table 5 (Continued)

\begin{tabular}{|c|c|c|c|c|}
\hline Antiretroviral drug & Comedication & $\begin{array}{l}\text { Frequency, n (\%) } \\
(n=194)\end{array}$ & $\begin{array}{l}\text { Strength of } \\
\text { recommendation }^{a}\end{array}$ & Quality of evidence ${ }^{b}$ \\
\hline & $\begin{array}{l}\text { Iron supplement } \\
\text { Itraconazole } \\
\text { Magnesium } \\
\text { Metformin } \\
\text { Midazolam } \\
\text { Nifedipine } \\
\text { Phenytoin } \\
\text { Quetiapine } \\
\text { Rifabutin } \\
\text { Rifampicin } \\
\text { Saxagliptin } \\
\text { Sildenafil } \\
\text { Tamsulosin } \\
\text { Trazodone } \\
\text { Valproate } \\
\text { Zolpidem }\end{array}$ & $\begin{array}{l}\text { I }(0.5) \\
\text { I }(0.5) \\
\text { I }(0.5) \\
2(1.0) \\
\text { I }(0.5) \\
\text { I }(0.5) \\
\text { I }(0.5) \\
\text { I }(0.5) \\
\text { I }(0.5) \\
\text { I }(0.5) \\
\text { I }(0.5) \\
\text { I }(0.5) \\
\text { I }(0.5) \\
\text { I }(0.5) \\
\text { I }(0.5) \\
5(2.6)\end{array}$ & $\begin{array}{l}\text { Potential interaction } \\
\text { Potential interaction } \\
\text { Potential interaction } \\
\text { Potential interaction } \\
\text { Potential interaction } \\
\text { Potential interaction } \\
\text { Do not coadminister } \\
\text { Do not coadminister } \\
\text { Potential interaction } \\
\text { Do not coadminister } \\
\text { Potential interaction } \\
\text { Potential interaction } \\
\text { Potential interaction } \\
\text { Potential interaction } \\
\text { Potential interaction } \\
\text { Potential interaction }\end{array}$ & $\begin{array}{l}\text { Very low } \\
\text { Very low } \\
\text { Very low } \\
\text { Very low } \\
\text { Very low } \\
\text { Very low } \\
\text { Very low } \\
\text { Very low } \\
\text { Low } \\
\text { Moderate } \\
\text { Very low } \\
\text { Very low } \\
\text { Very low } \\
\text { Very low } \\
\text { Very low } \\
\text { Very low }\end{array}$ \\
\hline RAL & $\begin{array}{l}\text { Calcium supplement } \\
\text { Iron supplement } \\
\text { Magnesium } \\
\text { Rifampicin }\end{array}$ & $\begin{array}{l}1(0.5) \\
2(1.0) \\
6(3.1) \\
2(1.0)\end{array}$ & $\begin{array}{l}\text { Potential interaction } \\
\text { Potential interaction } \\
\text { Potential interaction } \\
\text { Potential interaction }\end{array}$ & $\begin{array}{l}\text { Very low } \\
\text { Very low } \\
\text { Very low } \\
\text { Moderate }\end{array}$ \\
\hline
\end{tabular}

Notes: ${ }^{a}$ Do not coadminister: these drugs should not be coadministered; Potential interaction: potential clinically significantly interaction that is likely to require additional monitoring, alteration of drug dosage or timing of administration. ${ }^{b}$ Find more information on quality of evidence at the following website: https://www.hivdruginteractions.org/.

Abbreviations: 3TC, lamivudine; ATV, atazanavir; COBI, cobicistat; DRV, darunavir; DTG, dolutegravir; EFV, efavirenz; ETR, etravirine; EVG, elvitegravir; IDV, indinavir; INSTIs, integrase strand transfer inhibitors; LPV, lopinavir; NNRTIs, non-nucleoside reverse transcriptase inhibitors; NRTIs, nucleoside reverse transcriptase inhibitors; PIs, protease inhibitors; RAL, raltegravir; RPV, rilpivirine; RTV, ritonavir; TDF, tenofovir disoproxil fumarate; ZDV, zidovudine.

that in $2016(60.4 \%)$. However, the prescription rate of TAF/FTC, the two NRTIs in Genvoya, was $31.3 \%$ in 2017, which is likely to account for the decrease in the prescription rate of TDF/FTC in 2017. This result may be further explained by the differences in the efficacy and safety of the regimens containing TDF/FTC and TAF/ FTC for the management of HIV infection. In comparison to TDF/FTC-based regimens, TAF/FTC-based regimens have similar or better efficacy, and their use improved renal and bone health. ${ }^{31-33}$ According to the study by Sax et al, which employed treatment-naïve HIV-1-infected patients, high virological success rates (HIV-1 RNA $<50$ copies $/ \mathrm{mL}$ ) were achieved at week 48 in patients receiving both TAF/FTC/COBI/EVG (92\%) and TDF/FTC/COBI/ EVG (90\%). ${ }^{34}$ In the study by Mills et al on virologically suppressed HIV-1-infected patients, virological success (HIV-1 RNA $<50$ copies $/ \mathrm{mL}$ ) at week 48 occurred in $97 \%$ of the patients receiving $\mathrm{TAF} / \mathrm{FTC} / \mathrm{COBI} / \mathrm{EVG}$ and $93 \%$ of those administered with the TDF-containing regimen $(P<0.0002) .{ }^{35}$ In particular, TAF/FTC/COBI/EVG was preferred over TDF/FTC/COBI/EVG for HIV-1-infected patients who were aged $\geq 50$ years. ${ }^{33,34}$

The intracellular concentration of tenofovir-diphosphate, which is an active metabolite, was approximately four times higher after treatment with TAF than that after treatment with TDF. ${ }^{34}$ This indicated that compared with TDF, TAF is required at much lower doses, and the systemic exposure of tenofovir was also expected to be much lower in patients under therapy with TAF than in those treated with TDF. ${ }^{34}$ Consequently, this is likely to improve tenofovir-associated AEs such as renal toxicity and reduced bone mineral density (BMD). Sax et al reported that therapy with TAF/FTC/ $\mathrm{COBI} / \mathrm{EVG}$ induced a significantly smaller increase in mean serum creatinine ( 0.08 vs $0.12 \mathrm{mg} / \mathrm{dL} ; P<0.0001)$, significantly lesser proteinuria (median \% change, -3 vs 20 ; $P<0.0001$ ), and a significantly smaller decrease in the BMD of the spine (mean \% change, -1.30 vs $-2.86 ; P<0.0001$ ) and hip $(-0.66 \mathrm{vs}-2.95 ; P<0.0001)$ at week 48 than those observed after treatment with TDF/FTC/COBI/EVG. ${ }^{34}$ Mills et al additionally reported that compared with treatment with TDF-containing regimens, therapy with TAF/FTC/COBI/ EVG significantly improved the BMD of the spine (mean \% change from the baseline, $1.56 \mathrm{vs}-0.44 ; P<0.0001)$ and hip (1.47 vs $-0.34 ; P<0.0001)$ and the mean serum creatinine (-0.4 vs $2.9 \mu \mathrm{mol} / \mathrm{L} ; P<0.0001)$ at week $48 .{ }^{35}$ Comprehensively, TAF/FTC/COBI/EVG may be a better choice than TDF/FTC/COBI/EVG for the treatment of HIV-infected patients, especially those aged $\geq 50$ years, and patients who 
have reduced renal function, medical history of fractures, osteopenia, or osteoporosis.

Exposure to ABC, the NRTI present in Triumeq, may lead to an increase in the risks of CVD events, such as coronary artery disease and myocardial infarction. ${ }^{31,36}$ According to the study that assessed the risk of CVD events in HIV-infected patients administered with ARV drugs, a higher incidence rate of CVD events was observed in the patients who were currently exposed to $\mathrm{ABC}$ than in those who were currently exposed to other ARV drugs $(9.74 / 1,000$ person-years vs 5.75/1,000 person-years). ${ }^{36}$ The HRs of CVD events for patients under current $(1.43 ; P=0.001)$, recent (1.41; $P=0.001)$, and cumulative (1.18 [per year]; $P=0.002)$ exposure to $\mathrm{ABC}$ increased with statistical significance. ${ }^{36}$ The HR of CVD events for cumulative exposure to $\mathrm{ABC}$ also increased for up to 24 months and decreased thereafter. ${ }^{36}$ Consequently, ABC should be cautiously used in HIV-infected patients, especially those aged $\geq 50$ years, and patients with risk factors (such as HTN, hyperlipidemia, DM, and smoking) for coronary artery disease and myocardial infarction, by appropriately managing those risk factors prior to initiating regimens containing $\mathrm{ABC}$.

The DDIs between ART and non-ART drugs make it difficult to design effective and safe ART regimens, especially in older HIV-infected patients ( $\geq 50$ years of age), who are more likely to take one or more comedications with ART drugs in order to manage multiple comorbidities than younger HIV-infected patients $(<50$ years of age) are. ${ }^{4,37}$ The independently associated variables with potential or contraindicated DDIs include older age, dyslipidemia, higher daily drug burden of non-ARTs, and prescription of PIs. ${ }^{4}$ In this study, contraindicated drugs, such as alfuzosin, clopidogrel, quetiapine, rifampicin, simvastatin, and phenytoin, were frequently prescribed along with ARV regimens including pharmacokinetic boosters (ie, RTV and COBI). Potential DDIs mainly occurred between boosted ARV regimens and non-ART drugs, such as drugs prescribed for gastrointestinal, metabolic, cardiovascular, and central nervous system ailments, which was similar to the results obtained from previous studies. ${ }^{4,37}$ ARV regimens including pharmacokinetic boosters should be cautiously administered to poly-medicated patients. Other regimens including INSTIs (ie, DTG and RAL) are preferable in those cases. Additionally, comprehensive pharmacist-led medication review and intervention in HIV-positive patients, especially those under complex medication regimens, may reduce the incidence rates of AEs and DDIs, as demonstrated in a previous study. ${ }^{7}$
This study has some limitations which should be borne in mind while interpreting the results. All data pertaining to the prescribed medications including ART agents were retrospectively collected by reviewing the electronic medical charts of the patients. Therefore, it could not be confirmed whether the patients had actually partaken of the prescribed medication and whether the DDIs had actually occurred. This limitation may be solved by providing the patients with self-reporting questionnaires concerning medication adherence and DDIs in the future. It was difficult to determine when the comorbidities had occurred owing to the cross-sectional design of this study. The last limitation was the representativeness of the patients included in this study. Most of the patients were likely to be current residents of North Jeolla Province in South Korea; thus, it may be somewhat difficult to generalize the results of this study and extend them to the residents of other regions of South Korea. In order to overcome this shortcoming, it is necessary to collaborate with other hospitals in the near future. However, since studies on the age-wise usage pattern of ARVs and their DDIs with non-ARV drugs have been rarely conducted in Korea, this study is of significance and could aid the identification of more appropriate ARV drug regimens having few DDIs with non-ARVs in an aging population of PLWH in Korea.

\section{Conclusion}

The advent of combination ARTs has enabled PLWH to live up to older ages, causing these individuals to experience age-related illnesses, which necessitates the use of non-ARV medications for managing these illnesses. It is important to use appropriate ART regimens that are less likely to interact with other comedications and affect nonAIDS illnesses. The most frequently prescribed ART regimen involves treatment with NRTIs/INSTIs (ie, ABC/3TC/DTG, TAF/FTC/COBI/EVG, and TDF/FTC/COBI/EVG). TAF/ FTC/COBI/EVG may be a better option than TDF/FTC/ $\mathrm{COBI} / \mathrm{EVG}$ and $\mathrm{ABC} / 3 \mathrm{TC} / \mathrm{DTG}$ for patients, especially those aged $\geq 50$ years, and those having low BMD, reduced kidney function, or cardiovascular diseases. EVG/COBI and boosted PIs with RTV or COBI may not be good options for poly-medicated patients due to the high risks of DDIs, and DTG or RAL regimens may be preferred in this situation. However, EVG/COBI was most frequently prescribed in 2017. Further research should be performed to evaluate the impact of pharmacist-led medication review and intervention on AEs and DDIs in HIV-positive patients in Korea under complex medication regimens. 


\section{Acknowledgments}

This work was supported by the National Research Foundation of Korea grant funded by the Korea government (NRF2016R1C1B1015938).

\section{Disclosure}

The authors report no conflicts of interest in this work.

\section{References}

1. Oguntibeju OO. Quality of life of people living with HIV and AIDS and antiretroviral therapy. HIV AIDS. 2012;4:117-124.

2. Maggi P, Di Biagio A, Rusconi S, et al. Cardiovascular risk and dyslipidemia among persons living with HIV: a review. BMC Infect Dis. 2017;17(1):551

3. Mahy M, Autenrieth CS, Stanecki K, Wynd S. Increasing trends in HIV prevalence among people aged 50 years and older: evidence from estimates and survey data. AIDS. 2014;28(Suppl 4):S453-S459.

4. Holtzman C, Armon C, Tedaldi E, et al. Polypharmacy and risk of antiretroviral drug interactions among the aging HIV-infected population. J Gen Intern Med. 2013;28(10):1302-1310.

5. Lin CF, Wang CY, Bai CH. Polypharmacy, aging and potential drugdrug interactions in outpatients in Taiwan: a retrospective computerized screening study. Drugs Aging. 2011;28(3):219-225.

6. Secoli SR, Figueras A, Lebrão ML, de Lima FD, Santos JL. Risk of potential drug-drug interactions among Brazilian elderly: a populationbased, cross-sectional study. Drugs Aging. 2010;27(9):759-770.

7. McNicholl IR, Gandhi M, Hare CB, Greene M, Pierluissi E. A pharmacist-led program to evaluate and reduce polypharmacy and potentially inappropriate prescribing in older HIV-positive patients. Pharmacotherapy. 2017;37(12):1498-1506.

8. Cantudo-Cuenca MR, Jiménez-Galán R, Almeida-Gonzalez CV, Morillo-Verdugo R. Concurrent use of comedications reduces adherence to antiretroviral therapy among HIV-infected patients. $J$ Manag Care Spec Pharm. 2014;20(8):844-850.

9. Baecke C, Gyssens IC, Decoutere L, van der Hilst JCH, Messiaen P. Prevalence of drug-drug interactions in the era of HIV integrase inhibitors: a retrospective clinical study. Neth J Med. 2017;75(6):235-240.

10. Tseng A, Szadkowski L, Walmsley S, Salit I, Raboud J. Association of age with polypharmacy and risk of drug interactions with antiretroviral medications in HIV-positive patients. Ann Pharmacother. 2013; 47(11):1429-1439.

11. Bastida C, Grau A, Márquez M, et al. Polypharmacy and potential drugdrug interactions in an HIV-infected elderly population. Farm Hosp. 2017;41(5):618-624.

12. Smith JM, Flexner C. The challenge of polypharmacy in an aging population and implications for future antiretroviral therapy development. AIDS. 2017;31(Suppl 2):S173-S184.

13. Molas E, Luque S, Retamero A, et al. Frequency and severity of potential drug interactions in a cohort of HIV-infected patients identified through a multidisciplinary team. HIV Clin Trials. 2018;19(1):1-7.

14. Ghidei L, Simone MJ, Salow MJ, et al. Aging, antiretrovirals, and adherence: a meta analysis of adherence among older HIV-infected individuals. Drugs Aging. 2013;30(10):809-819.

15. AIDSinfo. Guidelines for the use of antiretroviral agents in adults and adolescents living with HIV. Available from: https://aidsinfo.nih.gov/ guidelines/html/1/adult-and-adolescent-arv/0. Accessed April 4, 2018.

16. World Health Organization. Consolidated guidelines on the use of antiretroviral drugs for treating and preventing HIV infection: recommendations for a public health approach - second edition; 2016. Available from: http://www.who.int/hiv/pub/arv/arv-2016/en/. Accessed April 4, 2018.

17. Liverpool HIV Drug Interactions [homepage on the Internet]. Available from: https://www.hiv-druginteractions.org/. Accessed September 6, 2018.
18. Escota GV, O’Halloran JA, Powderly WG, Presti RM. Understanding mechanisms to promote successful aging in persons living with HIV. Int J Infect Dis. 2018;66:56-64.

19. Güerri-Fernandez R, Vestergaard P, Carbonell C, et al. HIV infection is strongly associated with hip fracture risk, independently of age, gender, and comorbidities: a population-based cohort study. J Bone Miner Res. 2013;28(6):1259-1263.

20. Palella FJ, Baker RK, Moorman AC, et al. Mortality in the highly active antiretroviral therapy era: changing causes of death and disease in the HIV outpatient study. J Acquir Immune Defic Syndr. 2006; 43(1):27-34.

21. COMBIVIR ${ }^{\circledR}$. Package Insert. Available from: https://www.accessdata. fda.gov/drugsatfda_docs/label/2015/20857s0301bl.pdf. Accessed April 11, 2018.

22. Flynn PM, Rodman J, Lindsey JC, et al. Intracellular pharmacokinetics of once versus twice daily zidovudine and lamivudine in adolescents. Antimicrob Agents Chemother. 2007;51(10):3516-3522.

23. Ruane PJ, Richmond GJ, Dejesus E, et al. Pharmacodynamic effects of zidovudine $600 \mathrm{mg}$ once/day versus $300 \mathrm{mg}$ twice/day in therapy-naïve patients infected with human immunodeficiency virus. Pharmacotherapy. 2004;24(3):307-312.

24. STRIBILD ${ }^{\circledR}$. Package Insert. Available from: https://www.gilead. com/ /media/files/pdfs/medicines/hiv/stribild/stribild_pi.pdf?la=en. Accessed April 11, 2018.

25. GENVOYA ${ }^{\circledR}$. Package Insert. Available from: https://www.gilead. com/ /media/files/pdfs/medicines/hiv/genvoya/genvoya_pi.pdf?la=en. Accessed April 11, 2018.

26. TRIUMEQ ${ }^{\circledR}$. Package Insert. Available from: https://www. viivhealthcare.com/media/80846/Triumeq-PI-MG.pdf. Accessed April 11, 2018.

27. Gallant JE, Dejesus E, Arribas JR, et al. Tenofovir DF, emtricitabine, and efavirenz vs. zidovudine, lamivudine, and efavirenz for HIV. NEngl J Med. 2006;354(3):251-260.

28. Pozniak AL, Gallant JE, Dejesus E, et al. Tenofovir Disoproxil Fumarate, emtricitabine, and efavirenz versus fixed-dose zidovudine/ lamivudine and efavirenz in antiretroviral-naive patients: virologic, immunologic, and morphologic changes-a 96-week analysis. J Acquir Immune Defic Syndr. 2006;43(5):535-540.

29. Truong WR, Schafer JJ, Short WR. Once-daily, single-tablet regimens for the treatment of HIV-1 infection. P\&T. 2015;40(1):44-55.

30. Sebaaly JC, Kelley D. Single-tablet regimens for the treatment of HIV-1 infection. Ann Pharmacother. 2017;51(4):332-344.

31. Angione SA, Cherian SM, Özdener AE. A review of the efficacy and safety of Genvoya ${ }^{\circledR}$ (elvitegravir, cobicistat, emtricitabine, and tenofovir alafenamide) in the Management of HIV-1 infection. $J$ Pharm Pract. 2018;31(2):216-221.

32. Raffi F, Orkin C, Clarke A, et al. Brief Report: long-term (96-week) efficacy and safety after switching from tenofovir disoproxil fumarate to tenofovir alafenamide in HIV-infected, virologically suppressed adults. J Acquir Immune Defic Syndr. 2017;75(2):226-231.

33. Arribas JR, Thompson M, Sax PE, et al. Brief report: randomized, double-blind comparison of tenofovir alafenamide (TAF) vs tenofovir disoproxil fumarate (TDF), each coformulated with elvitegravir, cobicistat, and emtricitabine (E/C/F) for initial HIV-1 treatment: Week 144 results. J Acquir Immune Defic Syndr. 2017;75(2): 211-218.

34. Sax PE, Wohl D, Yin MT, et al. Tenofovir alafenamide versus tenofovir disoproxil fumarate, coformulated with elvitegravir, cobicistat, and emtricitabine, for initial treatment of HIV-1 infection: two randomised, double-blind, phase 3, non-inferiority trials. Lancet. 2015;385(9987): 2606-2615.

35. Mills A, Arribas JR, Andrade-Villanueva J, et al. Switching from tenofovir disoproxil fumarate to tenofovir alafenamide in antiretroviral regimens for virologically suppressed adults with HIV-1 infection: a randomised, active-controlled, multicentre, open-label, phase 3, noninferiority study. Lancet Infect Dis. 2016;16(1):43-52. 
36. Dorjee K, Baxi SM, Reingold AL, Hubbard A. Risk of cardiovascular events from current, recent, and cumulative exposure to abacavir among persons living with HIV who were receiving antiretroviral therapy in the United States: a cohort study. BMC Infect Dis. 2017;17(1):708.
37. Marzolini C, Back D, Weber R, et al. Ageing with HIV: medication use and risk for potential drug-drug interactions. J Antimicrob Chemother. 2011;66(9):2107-2111.

\section{Publish your work in this journal}

Therapeutics and Clinical Risk Management is an international, peerreviewed journal of clinical therapeutics and risk management, focusing on concise rapid reporting of clinical studies in all therapeutic areas outcomes, safety, and programs for the effective, safe, and sustained use of medicines. This journal is indexed on PubMed Central, CAS
EMBase, Scopus and the Elsevier Bibliographic databases. The manuscript management system is completely online and includes a very quick and fair peer-review system, which is all easy to use. Visit http://www.dovepress.com/testimonials.php to read real quotes from published authors.

Submit your manuscript here: http://www.dovepress.com/therapeutics-and-clinical-risk-management-journal 